\title{
Tsafon
}

Revue d'études juives du Nord

$81 \mid 2021$

Des synagogues à travers les âges Lieux de prières, lieux d'études et autres fonctions

\section{Jean Jülich, un homme (extra-) ordinaire}

\section{Carmen Grabuschnig}

\section{(2) OpenEdition}

Journals

Édition électronique

URL : https://journals.openedition.org/tsafon/3970

DOI : $10.4000 /$ tsafon.3970

ISSN : 2609-6420

Éditeur

Association Jean-Marie Delmaire

Édition imprimée

Date de publication : 1 juillet 2021

Pagination : 151-154

ISSN : $1149-6630$

\section{Référence électronique}

Carmen Grabuschnig, "Jean Jülich, un homme (extra-) ordinaire », Tsafon [En ligne], 81 | 2021, mis en ligne le 01 juillet 2021, consulté le 15 septembre 2021. URL : http://journals.openedition.org/tsafon/ 3970 ; DOI : https://doi.org/10.4000/tsafon.3970 


\title{
Hommage
}

\section{Jean Jülich, un homme (extra-) ordinaire}

\author{
Carmen Grabuschnig*
}

L'anniversaire des dix ans de son décès (19 octobre 2011) est l'occasion de rappeler l'histoire d'un homme dont la vie et le destin sont probablement inconnus au lecteur français et forcent l'admiration : celle de Jean Jülich. Colonais authentique et citoyen engagé, membre actif du carnaval de Cologne, musicien, restaurateur, "Pirate de l'edelweiss » opposé au national-socialisme, porteur de la Croix fédérale du mérite et Juste parmi les Nations : ces qualificatifs témoignent de la vie tourmentée et riche qu'a connue cet homme ainsi que de sa volonté et de sa détermination à la vivre selon son propre gré, en toute circonstance. Cependant, avant d'être reconnu pour ses engagements, Jean Jülich a été traité au cours de sa vie d'ennemi public, de criminel et d'asocial.

Cette histoire de Jean Jülich, qui a donné lieu à tant de polémiques, commence le 18 avril 1929. Fils d'une mère travaillant à l'usine et d'un père communiste obligé dès 1933 de vivre dans la clandestinité puis arrêté par les nationaux-socialistes en 1935, Jean Jülich grandit la majeure partie de son enfance chez ses grands-parents. En 1939, il rejoint la Jeunesse hitlérienne comme beaucoup d'autres jeunes de l'époque mais très vite le service dans la jeunesse d'État l'ennuie. «D'emblée j'ai eu une aversion contre le fait de se mettre au garde-à-vous, contre l'obéissance aveugle $»^{1}$, explique-t-il plus tard. Fin 1942/début 1943, il entre en contact avec d'autres adolescents partageant cette même aversion pour la Jeunesse hitlérienne : les «Pirates de l'edelweiss ». Ces groupes de jeunes, garçons

\footnotetext{
* Docteure en sociologie, École Pratique des Hautes Études/GSRL.

${ }^{1}$ Traduit de l'original : "Von Grund zu hab ich eine Aversion gegen Strammstehen, Kadavergehorsam. ». Entretien avec Jean Jülich réalisé par le centre de documentation sur le national-socialisme à Cologne, consultable sur :

http://www.eg.nsdok.de/default.asp?typ $=$ interview\&pid=47\&aktion=erstes, lien vérifié le $27 / 04 / 2021$.
} 
et filles, n'excédant pas pour la plupart la taille d'un cercle d'amis, existent dans un grand nombre de villes de l'ouest de l'Allemagne. Ils cultivent un style vestimentaire particulier et affichent leurs différences avec la Jeunesse hitlérienne également à travers leur musique et leur mode de vie.

Dans son autobiographie, Jean Jülich relate l'épisode de sa rencontre avec les «Pirates de l'edelweiss » : « Ces garçons et filles m'ont abordé de manière spontanée. Il émanait d'eux un côté non conformiste et romantique, pas la discipline autoritaire et bornée de la Jeunesse hitlérienne ${ }^{2}{ }^{2}$. Désormais, Jean Jülich retrouve régulièrement ces adolescents non conformistes, le soir à l'entrée du bunker où ils chantent leurs chansons, le weekend quand ils partent tous ensemble en randonnée aux alentours de Cologne.

L'aspiration de ces jeunes à vivre leur vie selon leur propre gré ne tarde pas à entrer en contradiction avec le projet totalitaire du régime national-socialiste. Des patrouilles de la Jeunesse hitlérienne les contrôlent sans cesse, des bagarres éclatent fréquemment. L'absence de convictions politiques chez les «Pirates de l'edelweiss » au début du mouvement se transforme pour certains en détermination à agir : Jean Jülich et ses amis concoctent des plans, ils expérimentent avec de la poudre noire et du carbure, ils construisent des catapultes mais leurs projets restent, selon les dires de Jean Jülich lui-même, au stade de la simple rêverie.

Grâce à Barthel Schink et Bubbes Rheinberger, des amis, également "Pirates de l'edelweiss », Jean Jülich lie des contacts avec le groupe Steinbrück en 1944. Ce groupe, composé de déserteurs, de prisonniers de guerre, d'opposants politiques, de «Pirates de l'edelweiss » et de délinquants, s'est constitué dans la clandestinité et organise des pillages afin d'assurer la survie de ses membres. La maison de Hans Steinbrück dans le quartier d'Ehrenfeld (à Cologne) sert de point de rencontre et de dépôt d'armes, rassemblées, selon Jean Jülich, dans le but de faire exploser la centrale de la Gestapo à Cologne. À plusieurs reprises, des Juifs trouvent refuge dans le sous-sol de l'appartement de la Schönsteinstrasse. Fin septembre/début octobre 1944, le groupe Steinbrück est démantelé : treize de ses membres, dont les deux amis de Jean Jülich, sont exécutés publiquement le 10 novembre 1944. Jean Jülich est lui-même arrêté en octobre 1944, interrogé par la Gestapo, torturé puis incarcéré sans

\footnotetext{
${ }^{2}$ Traduit de l'original : « Diese Jungen und Mädchen sprachen mich spontan an. Sie strahlten etwas Unangepasstes und Romantisches aus, nicht den autoritären und engstirnigen Drill der Hitlerjugend ». Jean Jülich, Kohldampf, Knast und Kamelle - Ein Edelweißpirat erzählt sein Leben, Cologne, Verlag Kiepenheuer \& Witsch, 2003, p. 44.
} 
jugement. Il n'est libéré par les troupes américaines qu'en 1945 et sort affaibli et typhoïdique.

Après la guerre, Jean Jülich travaille d'abord comme kiosquier, puis comme restaurateur. Il devient également participant actif du carnaval de Cologne. Dans un premier temps, son passé comme «Pirate de l'edelweiss » passe au second plan face aux nécessités du quotidien. Quand, à la fin des années soixante-dix, une discussion publique est déclenchée suite au refus de l'Organisme d'indemnisation des victimes d'accorder le statut de persécuté du régime à Barthel Schink, Jean Jülich ne peut, une fois de plus, s'empêcher de prendre position : il est obligé de défendre la mémoire de son ami. Dans la « Controverse de Cologne $»^{3}$, les événements d'Ehrenfeld et les «Pirates de l'edelweiss » sont désormais amalgamés et Barthel Schink devient le symbole emblématique d'une lutte acharnée opposant deux camps adverses, les tenants de la thèse de la criminalité et les défenseurs de la thèse de la résistance.

L'attribution du titre de Juste parmi les Nations à Jean Jülich, Michael Jovy ${ }^{4}$ et Bartholomäus Schink en 1984 fait de nouvelles vagues : cette attribution est le fruit du travail du journaliste et écrivain Peter Finkelgruen qui, apprenant l'histoire des "Pirates de l'edelweiss », se lance dans des recherches et remet en 1981 le résultat de ce travail à Yad Vashem. À cette époque, Peter Finkelgruen vit en Israël où il travaille pour la Friedrich-Naumann-Stiftung. Le parlement du Land de Rhénanie-duNord-Westphalie se voit confronté à la demande de revoir le statut des « Pirates de l'edelweiss » dans la mémoire officielle pour qu'ils soient enfin reconnus comme résistants ; une expertise est commandée. L'étude de l'historien Bernd A. Rusinek conclut certes que ces jeunes ne peuvent être qualifiés de criminels mais leur nie tout statut de résistant : pour lui, parler de résistance nécessiterait une mentalité éthiquement élevée, laquelle leur aurait fait défaut ${ }^{5}$. Jean Jülich, comme beaucoup d'autres anciens membres de ces groupes, est déçu par cette conclusion. Ce qui le

\footnotetext{
${ }^{3}$ Nom générique par lequel l'historiographie se réfère aujourd'hui aux discussions de l'époque. Un très bon résumé des différentes étapes de cette controverse se trouve dans : Martin Rüther, "Senkrecht stehen bleiben », Wolfgang Ritzer und die Edelweißpiraten, Unangepasstes Jugendverhalten im Nationalsozialismus und dessen späte Verarbeitung, Cologne, Emons Verlag, 2015, p. 162-179.

${ }^{4}$ Résistant et leader d'un groupe de jeunesse sous le Troisième Reich. Cf. Ansgar S. Klein, Michael Jovy dans : Internetportal Rheinische Geschichte, consultable sur : http://www.rheinische-geschichte.lvr.de/Persoenlichkeiten/michael-jovy/DE2086/lido/57c92fc064f8d4.40102215, lien vérifié le 27/04/2021.

${ }^{5} \mathrm{Cf}$. Bernd A. Rusinek, Gesellschaft in der Katastrophe, Terror, Illegalität, Widerstand, Köln 1944/45, Essen, Klartext Verlag, 1989.
} 
dérange avant tout, c'est le refus obstiné de Bernd A. Rusinek de prendre en compte les récits de ceux qui avaient été impliqués dans les événements. En 2003, il décide donc de publier son autobiographie, donne des conférences et raconte son histoire dans les écoles. En tant que musicien passionné, il contribue à des projets de transmission du répertoire musical des «Pirates de l'edelweiss » aux jeunes générations (enregistrements, participation au festival des "Pirates de l'edelweiss »). En un mot, il participe activement au travail de mémoire concernant ces groupes de jeunes ayant défié le projet national-socialiste d'une jeunesse mise au pas.

« Nous avons été des jeunes gens, nous avons instinctivement eu le sentiment que quelque chose n'allait pas! Qu'on n'était pas pour! ${ }^{6}$, expliquait Jean Jülich. Qu'importe dès lors que ce sentiment n'ait pas été théorisé à l'époque ! Les « Pirates de l'edelweiss » ont su créer un espace où l'altérité pouvait être vécue; ils s'opposaient de manière directe au projet totalitaire du régime national-socialiste. Jean Jülich et ses amis, s'ils n'arrivèrent pas toujours à mettre en œuvre leurs projets de résistance ${ }^{7}$, ont participé à la création de cet espace, ils ont attaqué le régime nationalsocialiste là où il était atteignable, ils ont aidé ceux qui étaient persécutés. Nul ne l'a mieux formulé que Jan Krauthäuser lors du décès de son ami :

Avec Jean Jülich nous avons perdu un citoyen engagé et courageux ainsi qu'un Colonais authentique! Nous lui devons beaucoup: en tant que «Pirate de l'edelweiss », en tant que pionnier infatigable d'un travail de mémoire équitable, en tant que participant au carnaval, en tant qu'artiste, en tant qu'inspirateur... ! [...] Nous le commémorerons en chantant, en faisant des randonnées et en combattant pour l'équité. ${ }^{8}$

\footnotetext{
${ }^{6}$ Traduit de l'original : « Wir waren junge Menschen, hatten das Gefühl aus dem Bauch raus, das stimmt nit ! Da sind wir nicht für ! » Entretien avec Jean Jülich, op. cit.

${ }^{7}$ Comme l'écrit très bien le grand historien français de la résistance, Jean-Pierre Azéma : «La mémoire de la résistance se doit d'être glorieuse... comme si résister avait consisté exclusivement à faire sauter des pylônes et des trains ou à peupler les maquis des héros porteurs de mitraillettes Sten » Jean-Pierre Azéma, « Préface » à Jacques Semelin, Sans armes face à Hitler, La résistance civile en Europe (1939-1943), Paris, Éditions Payot, 1989, p. 7-8.

${ }^{8}$ Traduit de l'original : « Mit Jean Jülich haben wir zugleich einen sehr engagierten, couragierten Bürger wie ein echtes Kölsches Original verloren! Wir verdanken ihm viel: als Edelweißpirat, als unermüdlicher Vorkämpfer für eine gerechte Vergangenheitsbewältigung, als Karnevalist, als Künstler, als Inspirator...! (...) Wir werden ihm gedenken, wenn wir singen, wenn wir wandern und wenn wir für Gerechtigkeit kämpfen! » Cité après Roland Kaufhold, «"E Hätz so jroß wie ne Stään” - Zum Tode von Jean Jülich », consultable sur https://www.hagalil.com/2011/10/juelich/, lien vérifié le 27/04/2021.
} 